\title{
Impact of cigarette smoke and IL-17A activation on asthmatic patients with chronic rhinosinusitis*
}

\author{
Chien-Chia Huang ${ }^{1,2 \#}$, Ta-Jen Lee ${ }^{1,3 \#}$, Chi-Che Huang ${ }^{1,2}$, Po-Hung Chang 1,2, \\ Chia-Hsiang Fu',2, Pei-Wen Wu', ${ }^{1,4}$, Chun-Hua Wang ${ }^{5}$ \\ ' Division of Rhinology, Department of Otolaryngology, Chang Gung Memorial Hospital and Chang Gung University, Taoyuan, \\ Taiwan \\ ${ }^{2}$ Graduate Institute of Clinical Medical Sciences, College of Medicine, Chang Gung University, Taiwan \\ ${ }^{3}$ Department of Otolaryngology, Xiamen Chang Gung Hospital, Xiamen, China \\ ${ }^{4}$ Department of Otolaryngology-Head and Neck Surgery, Chang Gung Memorial Hospital and Chang Gung University, Keelung, \\ Taiwan \\ Rhinology 57: 1, 57 - 66, 2019 \\ https://doi.org/10.4193/Rhin18.131 \\ *Received for publication: \\ July 9, 2018 \\ Accepted: September 4, 2018 \\ ${ }^{5}$ Department of Thoracic Medicine, Chang Gung Memorial Hospital and Medicine of College, Chang Gung University, Taoyuan, \\ \# These authors contributed equally \\ Taiwan
}

Background: Cigarette smoke have adverse effects in the control of asthma and chronic rhinosinusitis (CRS). Interleukin (IL)-17A, the signature cytokine of helper T 17 cells and group 3 innate lymphoid cells (ILC3), has been reported to link with resistance to therapy in airway inflammation. This study aimed to investigate the impact of cigarette smoking and IL-17A activation on the clinical outcomes of asthmatic patients with chronic rhinosinusitis.

Methods: 33 patients with CRS and asthma, including 15 smokers and 18 non-smokers, and 7 asthmatic patients without CRS and smoking were prospectively recruited. The Sino-Nasal Outcome Test-22 and Asthma Control Test were used to evaluate sinonasal symptoms and the level of asthma control, respectively. Real-time PCR and immunostaining were applied to evaluate the expression levels of IL-17A and associated immunological factors on surgically-obtained nasal tissues.

Results: Nasal surgery improved both sinonasal symptoms and asthma control. Compared to non-smokers, smokers showed poorer improvement in asthma control. The expression of IL-17A, IL-22, aryl hydrocarbon receptor (AhR), and ILC3 was increased in the nasal tissues of smokers with asthma and CRS. The expression of IL-17A mRNA was correlated with that of AhR and with positive nuclear AhR-AhR nuclear translocator staining cells, and that of cyclooxygenase-2 enzyme (COX-2). ILC3 cells were associated with IL-17A, IL-22, AhR, and COX-2 mRNA expression.

Conclusions: Cigarette smoking was related to lesser improvement in asthma control after nasal surgery and to IL-17A activation in the nasal tissues of patients with inflammatory airways.

Key words: aryl hydrocarbon receptor, asthma, cigarette smoking, chronic rhinosinusitis, group 3 innate lymphoid cell, interleukin-17A

\section{Introduction}

Chronic rhinosinusitis (CRS) is frequently associated with asthma ${ }^{(1)}$, and its presence is related to asthma severity. Among patients with asthma and CRS, pulmonary function and asthma control were closely related to the severity of nasal symptoms ${ }^{(2)}$. Exposure to cigarette smoke has been linked to a higher prevalence and severity of $\mathrm{CRS}^{(3-5)}$, contributing to increased nasal congestion, irritation, and airflow resistance. Several studies demonstrated that exposure to smoking diminishes long-term post-operative improvement of quality of life, delays olfactory recovery, and increases the risk of revision surgery after endoscopic sinus surgery (ESS) $)^{(6,7)}$.

Although the type 2 inflammatory cytokines are considered as major drivers of airway inflammation in patients with CRS and 
asthma ${ }^{(8,9)}$, interleukin (IL)-17A has been linked to neutrophil recruitment, mucosa remodeling, and resistance to corticosteroidbased therapy in airway diseases ${ }^{(10-12)}$. Helper T (Th) 17 cells are effector T-cell subsets and secrete pro-inflammatory cytokines such as IL-17A, IL-17F, and IL-22, which is implicated in the pathogenesis of several inflammatory diseases ${ }^{(13)}$. Group 3 innate lymphoid cells (ILC3s) have recently emerged as one of the producers of IL-17A $\mathrm{A}^{(14)}$, are prevalent at mucosal sites, and protect mucosal surfaces ${ }^{(15)}$. In an animal study, ILC3 cell-producing IL-17A facilitated the development of airway hyperreactivity ${ }^{(16)}$. Smokers with asthma develop neutrophilic airway inflammation, which is related to cigarette smoke-induced production of IL-17A together with other environmental stimuli acting on airway epithelial cells that induce neutrophil chemotaxis ${ }^{(17)}$. Our recent study demonstrated that current smoking was associated with the augmented expression of IL-17A in the nasal tissues and with an increase in the number of unexpected emergency clinic visits in patients with asthma ${ }^{(18)}$. Thus, IL-17A plays a crucial role in cigarette smoke-induced airway inflammation and its resistance to the current therapeutic regimens.

Aryl hydrocarbon receptor (AhR), a ligand-activated transcription factor that modulates immune reactions, responds to 2,3,7,8-tetrachlorodibenzo-p-dioxin (TCDD; dioxin) and other related polycyclic aromatic hydrocarbons (PAHs) ${ }^{(19)}$. After binding to ligand, AhR translocates to the nucleus and forms a heterodimer with the AhR nuclear translocator (ARNT). This AhR-ARNT complex binds to a dioxin-responsive element and initiates the transcription of genes that comprise the AhR gene battery, including cytochrome P450 family and cyclooxygenase-2 enzymes $(\mathrm{COX}-2)^{(20,21)}$. PAH is an important constituent of cigarette smoke that triggers AhR activation ${ }^{(22,23)}$. Activation of AhR may be essential for the differentiation of ILC 3 and Th17 cells, as well as the consequent IL-17A-mediated immune response ${ }^{(24,25)}$. Therefore, we hypothesise that AhR-, ILC3-, and IL-17A-mediated immune responses may play a crucial role in cigarette smoke- related airway inflammation and its resistance to the current therapeutic regimens. Thus, this study aimed to investigate the impact of cigarette smoking on the clinical outcomes of asthmatic patients with CRS and its association with IL-17A activation.

\section{Materials and methods}

\section{Patients}

We prospectively recruited patients with CRS and asthma who were managed in the Departments of Thoracic Medicine and Otolaryngology between August 2013 and June 2016. Patients with asthma who fulfilled the diagnostic criteria of the Global Initiative for Asthma (GINA) guidelines ${ }^{(26)}$ and had as a co-morbidity, CRS (defined by the criteria from the European position paper ${ }^{(27)}$ and based on the symptoms and findings of nasal endoscopy and sinus computed tomography (CT)), were enrolled in the study. Patients with asthma [1] with regular follow-up for at least 1 year; [2] who have failed a 3-month maximal medical treatment, including intranasal topical corticosteroids, antihistamines, and broad-spectrum oral antibiotics; and [3] who planned to receive an ESS for CRS were included in the study. Patients with asthma were treated based on the GINA guidelines to achieve well-controlled status for more than 6 months before the surgery, that allowed them to undergo nasal surgery under general anaesthesia. Patients with previous nasal surgeries or major medical disorders, such as diabetes, nephrotic diseases, autoimmune disorders, immunodeficiencies, malignancies, and other chronic illnesses, were excluded. Another seven asthmatic patients without CRS (confirmed by sinus $C T$ ) and smoking were enrolled as a control group during the performance of septomeatoplasty and/or turbinoplasty for nasal obstruction. The study was approved by the institutional review board of the Chang Gung Memorial Hospital (IRB number: 101-5069B and 103-7085B). All participants signed an informed consent before enrolment.

The patients' clinical characteristics were recorded. They were

Table 1. Primer sequences specific to target genes.

\begin{tabular}{lcc} 
& \multicolumn{1}{c}{ Forward primers } & Reverse primers \\
\hline IL-4 & TTTGCTGCCTCCAAGAACACA & TCCTGTCGAGCCGTTTCAG \\
\hline IL-12A & TTCACCACTCCCAAAACCTG & AATGGTAAACAGGCCTCCAC \\
\hline IL-17A & TTGGTGTCACTGCTACTGCT & TTGGGCATCCTGGATTTCGT \\
\hline IL-22 & AGCCCTATATCACCAACCGC & TCTCCCCAATGAGACGAACG \\
\hline AhR & TTGTGCCGAGTCCCATATCC & TGGCAGGAAAAGGGTTGTT \\
AhRR & CTCCCAGACCTTTAGATGCG & CTTGGGAGTTTTGTTGCC \\
\hline CYP1A1 & CATTCAGGGAAGGGTTGGGTA \\
COX-2 & CTGGCACTTTAACCCCTACA & CACTCCCGCAACAGGAGT \\
\hline GADPH & ATAACGTGAAGGGCTGTCCC & CACACATGGG \\
\hline
\end{tabular}

IL, interleukin; AhR, Aryl hydrocarbon receptor;AhRR:aryl hydrocarbon receptor repressor; CYP1A1:cytochrome P450 family 1 subfamily A member 1; COX2, cyclooxygenase-2 enzymes; GAPDH, glyceraldehyde-3-phosphate dehydrogenase. 
asked if they regularly used cigarettes or smoked other forms of tobacco pre-operatively. Patients were considered as smokers if they reported regular or on-going smoking in the last 12 months ${ }^{(7)}$ and non-smokers if they never used cigarette regularly.

All patients received a thorough nasal endoscopy examination and sinus $\mathrm{CT}$, which were scored using the Lund-Kennedy endoscopy score and Lund-Mackay CT score preoperatively. Pulmonary function tests, including forced vital capacity (FVC), forced expiratory volume in 1 second $\left(\mathrm{FEV}_{1}\right)$, and $\mathrm{FEV}_{1} / \mathrm{FVC}$ ratio, were measured before and 6 months after nasal surgery. The Sino-Nasal Outcome Test-22 (SNOT-22) and Asthma Control Test (ACT) questionnaires were applied in order to evaluate the severity of sinonasal symptoms and the level of asthma control at the same time.

\section{Collection and processing of specimens}

Nasal mucosal specimens were rinsed in phosphate-buffered saline $(\mathrm{pH} 7.6)$, stored at liquid nitrogen at $-70^{\circ} \mathrm{C}$, and then processed for immunostaining and real-time polymerase chain reaction $(\mathrm{PCR})$.

\section{RNA extraction/reverse transcription and real-time PCR} Frozen nasal tissue was homogenised (Retsch, Haan, Germany). Total RNA was extracted from nasal specimens using the RNeasy Mini Kit (Qiagen $\mathrm{GmbH}$, Strasse, Germany) and quantified by NanoDrop (Thermo Scientific, Barrington, III). Reverse transcription was performed with random hexamer primers using the HighCapacity cDNA Reverse Transcription Kit (Applied Biosystems, Foster City, CA, USA). Real-time PCR was done using the TaqMan assay with primers specific for the target genes (Table S1) and glyceraldehyde-3-phosphate dehydrogenase (GAPDH) on the Applied Biosystems 7500 Fast Real-Time PCR System (Applied Biosystems). The amplification conditions consisted of an initial incubation at $95^{\circ} \mathrm{C}$ for $10 \mathrm{~min}$; followed by 45 cycles of $95^{\circ} \mathrm{C}$ for $10 \mathrm{~s}, 60^{\circ} \mathrm{C}$ for $20 \mathrm{~s}$, and $72^{\circ} \mathrm{C}$ for $10 \mathrm{~s}$; and a final cooling to $40^{\circ} \mathrm{C}$. Each sample was run in triplicate. The mean threshold cycle $(\mathrm{Ct})$ values were normalised to GAPDH, and the relative mRNA levels of the target genes were analysed using the $\Delta \Delta \mathrm{Ct}$ method.

\section{Immunohistochemistry for IL-17A expression}

Paraffin sections were de-waxed in xylene and rinsed in absolute alcohol, and incubated in $3 \% \mathrm{H}_{2} \mathrm{O}_{2}$ for 30 min to quench the endogenous peroxidase. The sections were microwaved and incubated in $0.2 \%$ normal swine serum (DAKO, CA, USA). The sections were incubated with the specific IL-17A antibody (diluted 1:100) or non-specific purified rabbit lgG (diluted 1:100) for $1 \mathrm{~h}$ (LifeSpan BioScience, WA, USA) and then used the avidin-biotin complex method (Vector Laboratories, CA, USA) for antibody labeling.
Immunostaining and confocal laser microscopic analysis Nasal tissues from paraffin sections were spun down on slide and fixed in methanol at $-20^{\circ} \mathrm{C}$. The cells were then blocked with $1 \% \mathrm{BSA} / \mathrm{PBS}$ at room temperature and incubated with the primary mouse anti-human antibody followed by anti-mouse antibody. Secondary antibodies with Alexa Fluor 488 and Alexa Fluor 594 conjugates (Invitrogen) were used in the detection of AhR (Santa Cruz) and ARNT (cell signaling), respectively. To study the natural-cytotoxicity-receptor-positive (NCR+) ILC3 cells, FITC-, $\mathrm{PE}-$, and Cy3-conjugated secondary antibodies (Sigma-Aldrich, Stockholm, Sweden) were used to detect CD127, NCR2, and IL-23R (Molecular Probes, Eugene, Ore), respectively. The nucleus was stained with DAPI (Santa cruz). The cells were mounted with anti-fade mounting medium (Dako Cytomation). Images were taken using a confocal laser-scanning microscope (Leica) and analysed using MetaMorph Image Analysis (Universal Imaging). Positively stained cells in merged images were counted in three high-power fields and presented as averaged cell numbers in each $\mathrm{mm}^{3}$.

\section{Statistical analyses}

The data were presented as mean \pm standard error and statistically analysed using the GraphPad Prism 5 (GraphPad Prism Software, Inc, San Diego, CA, USA). Categorical variables were compared using the $\chi^{2}$ test or Fisher's exact test, as appropriate. Continuous variables were analysed using the Mann-Whitney $\mathrm{U}$ test or Kruskal-Wallis $\mathrm{H}$ test when comparing between two or three groups. Perioperative changes were evaluated using a paired t-test or Wilcoxon signed-ranked test. The correlation was determined using the Spearman's correlation coefficient. Statistical significance was set at $p<0.05$.

\section{Results}

\section{Clinical characteristics of the study population}

The general characteristics of patients with asthma and CRS, including 15 smokers and 18 non-smokers, and 7 non-smokers with asthma without CRS are summarised in Table S2. There was no difference in age, severity of sinusitis, presence of nasal polyps, lung function, and medications between smokers and non-smokers with asthma and CRS, except for gender. Nasal polyps were found by nasal endoscopic examination in two of 15 smokers with asthma and CRS (13.3\%) and in six of 18 nonsmokers with asthma and CRS (33.3\%).

\section{Clinical outcomes after nasal surgery}

Nasal surgery improved sinonasal symptoms (Figure 1A), asthma control (Figure 1B), asthma treatment (Figure 1C), and pulmonary function (Figure 1D). Compared to non-smokers, smokers had poorer improvement in asthma control (Figure 1E), pulmonary function (Figure 1F), and asthma treatment (Figure 1G), but not in nasal symptoms (Figure 1H) after nasal surgery for CRS. 
Table S2. Clinical characteristics of the study participants.

\begin{tabular}{|c|c|c|c|c|}
\hline & \multicolumn{2}{|c|}{ Asthma and CRS } & \multirow{2}{*}{$\begin{array}{c}\text { Asthma } \\
\text { Non-smoker }(n=7)\end{array}$} & \multirow[t]{2}{*}{ P value* } \\
\hline & Smoker (n=15) & Non-smoker $(n=18)$ & & \\
\hline Age, years & $57.7 \pm 3.9$ & $52.8 \pm 2.8$ & $61.8 \pm 4.3$ & 0.28 \\
\hline Male : Female (n) & $14: 1$ & $4: 14$ & $5: 2$ & $<0.01$ \\
\hline Atopy(n) & 8 & 11 & 3 & 0.70 \\
\hline Nasal polyp (n) & $2(13.3 \%)$ & $6(33.3 \%)$ & 0 & $0.18+$ \\
\hline CT score & $8.4 \pm 1.1$ & $11.6 \pm 1.8$ & $0.0 \pm 0.0$ & $0.16+$ \\
\hline Endoscopy score & $4.0 \pm 0.9$ & $5.1 \pm 0.9$ & $1.0 \pm 0.4$ & $0.46+$ \\
\hline Serum IgE (IU/ml) & $421.9 \pm 211.7$ & $633.9 \pm 281.0$ & $405.9 \pm 180.7$ & 0.81 \\
\hline WBC (1000/uL) & $7.2 \pm 0.4$ & $9.2 \pm 1.1$ & $7.2 \pm 0.6$ & 0.36 \\
\hline Eosinophil (\%) & $2.4 \pm 0.5$ & $4.3 \pm 1.1$ & $5.0 \pm 1.6$ & 0.58 \\
\hline SNOT-22 & $59.9 \pm 8.5$ & $54.8 \pm 6.3$ & $87.6 \pm 11.9$ & 0.50 \\
\hline $\mathrm{ACT}$ & $19.9 \pm 1.3$ & $19.5 \pm 1.5$ & $19.4 \pm 3.1$ & 0.70 \\
\hline FVC (\% predicted) & $78.6 \pm 4.9$ & $70.7 \pm 4.0$ & $75.8 \pm 10.1$ & 0.62 \\
\hline FEV1 (\% predicted) & $71.7 \pm 4.8$ & $60.1 \pm 4.2$ & $59.7 \pm 7.0$ & 0.32 \\
\hline FEV1/FVC (\%) & $75.9 \pm 4.5$ & $74.6 .0 \pm 4.6$ & $77.1 \pm 3.5$ & 0.81 \\
\hline Anti-asthma medication ( $\mathrm{n}$ ) & & & & 0.56 \\
\hline Step 1 (as-needed SABA) & 0 & 0 & 0 & \\
\hline Step 2 (low dose ICS) & 4 & 4 & 1 & \\
\hline Step 3 (low dose ICS + LABA) & 6 & 7 & 2 & \\
\hline Step 4 (medium/high dose ICS + LABA ) & 3 & 0 & 1 & \\
\hline Step 5 (anti-IgE) & 2 & 7 & 3 & \\
\hline
\end{tabular}

Data are represented as mean \pm SE. CRS, chronic rhinosinusitis; CT, computed tomography; IgE, immunoglobulin E; WBC, white blood cell; SNOT-22, sino-nasal outcome test-22; ACT, asthma control test; FVC, forced vital capacity; FEV1, forced expiratory volume in 1 s; L, liter; SABA, short-acting beta2-agonist; ICS, inhaled corticosteroid; LABA, long-acting beta2-agonist. *Categorical variables were compared using Fisher's exact test and continuous variables were analyzed using the Mann-Whitney $\mathrm{U}$ test or Kruskal-Wallis $\mathrm{H}$ test when comparing between two or three groups. $+\mathrm{Analyses}$ between smoker and non-smoker with CRS and asthma.

Expressions of Th17-, Th1-, and Th2-related cytokines in the nasal tissues

Real-time PCR analysis of nasal tissues derived from smokers with asthma and CRS $(n=15)$ revealed a greater expression of IL-17A, IL-22 mRNA (signature cytokines of Th17 and ILC3 cells), AhR (transcription factor for IL-17A), aryl hydrocarbon receptor repressor (AhRR), cytochrome P450 family 1 subfamily A member 1 (CYP1A1, AhR target gene), and COX-2 (IL-17A-induced inflammatory mediators) than those of non-smokers with asthma and CRS $(n=18)$ or asthma without CRS $(n=7)$ (Figure 2AF). There were no statistical differences in the IL-4 (Th2-driven cytokine) and IL-12A (Th1-driven cytokine) mRNA expression between smokers and non-smokers (Figure $2 \mathrm{G}$ and $\mathrm{H}$ ). Immunohistochemistry studies of nasal tissues revealed a stronger expression of IL-17A in the epithelium, the endothelium of small vessels and inflammatory cells, and the mucus glands in the subepithelial tissues of smokers with asthma and CRS (Figure S1). Compared to smokers with asthma and CRS, non-smokers with asthma with or without CRS had a very weak expression of IL-17A in the epithelium, and this expression is absent in the endothelium, mucus glands, and subepithelial tissues.

\section{AhR-ARNT complex and natural cytotoxicity receptor group} 3 ILCs (NCR+ ILC3s) in biopsies

Nuclear AhR-ARNT complex was found in most smokers with asthma and CRS to a variable extent (Figure $3 \mathrm{~A}$ ). The number of positive cells associated with nuclear AhR-ARNT complex were significantly higher in smokers with asthma and CRS (24.6 \pm 2.6 $\times 10^{4}$ cells $\left./ \mathrm{mm}^{3}, \mathrm{n}=15\right)$ compared with that of non-smokers with asthma and CRS $\left(10.9 \pm 1.4 \times 10^{4}\right.$ cells $\left./ \mathrm{mm}^{3}, \mathrm{n}=18, \mathrm{p}<0.0001\right)$ and non-smokers with asthma in absence of CRS (12.2 $\pm 2.2 \times$ $10^{4}$ cells $/ \mathrm{mm}^{3}, \mathrm{n}=7, \mathrm{p}=0.006$ ) (Figure 3B). AhR nuclear localisation was not limited to the airway epithelium, but was also seen in the subepithelial cells including the inflammatory cells (Figure 3A). The number of AhR-ARNT positive cells was highly correlated to the level of IL-17 mRNA expression in the nasal tissues 

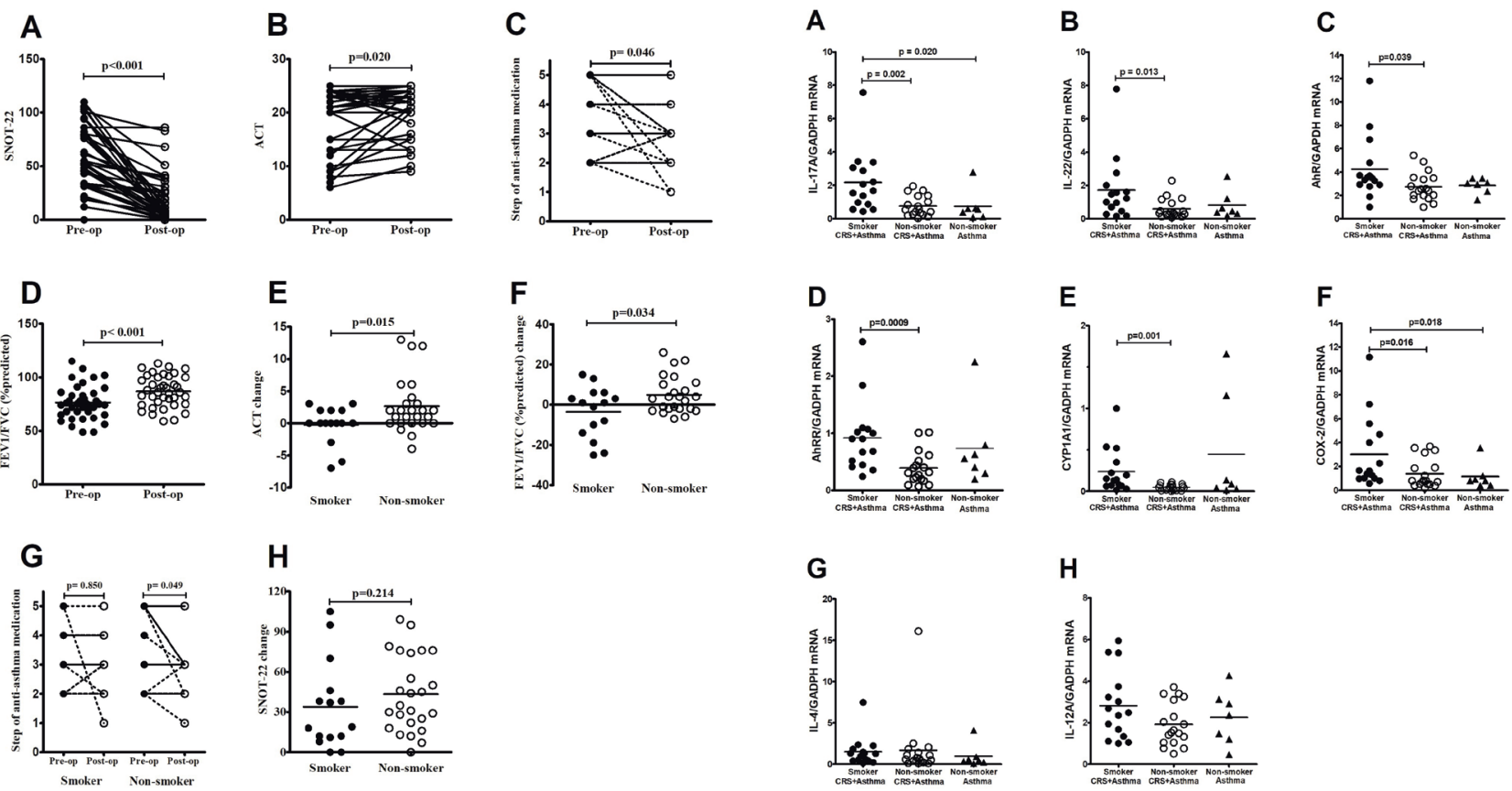

Figure 1. Nasal surgery improved sinonasal symptoms (A), asthma control (B), anti-asthma medication (C) and pulmonary function (D). Compared to the non-smokers, the smokers showed poorer improvement in asthma control (E) pulmonary function (F), and anti-asthma medication $(\mathrm{G})$ but not in nasal symptoms $(\mathrm{H})$ after nasal surgery for CRS. The significance is indicated. Pre-op=preoperative, Post$\mathrm{op}=$ postoperative.

Figure 2. The levels of IL-17A, IL-22, AhR, AhRR, CYP1A1, COX-2, IL-4 and IL-12A mRNA expression in nasal tissues. The mRNA expression of IL-17A (A), IL-22 (B) AhR (C), AhRR (D), CYP1A1 (E) and COX-2 (F) was significantly up-regulated in asthmatic patients with CRS who were smokers $(n=15)$ compared to asthmatic patients with CRS who were non-smokers $(n=18)$ or asthmatic patients without CRS who were non-smokers $(n=7)$. The mRNA levels of IL-4 $(\mathrm{G})$ and IL-12A $(\mathrm{H})$ in nasal tissues were not different between these three groups. The significance is indicated.

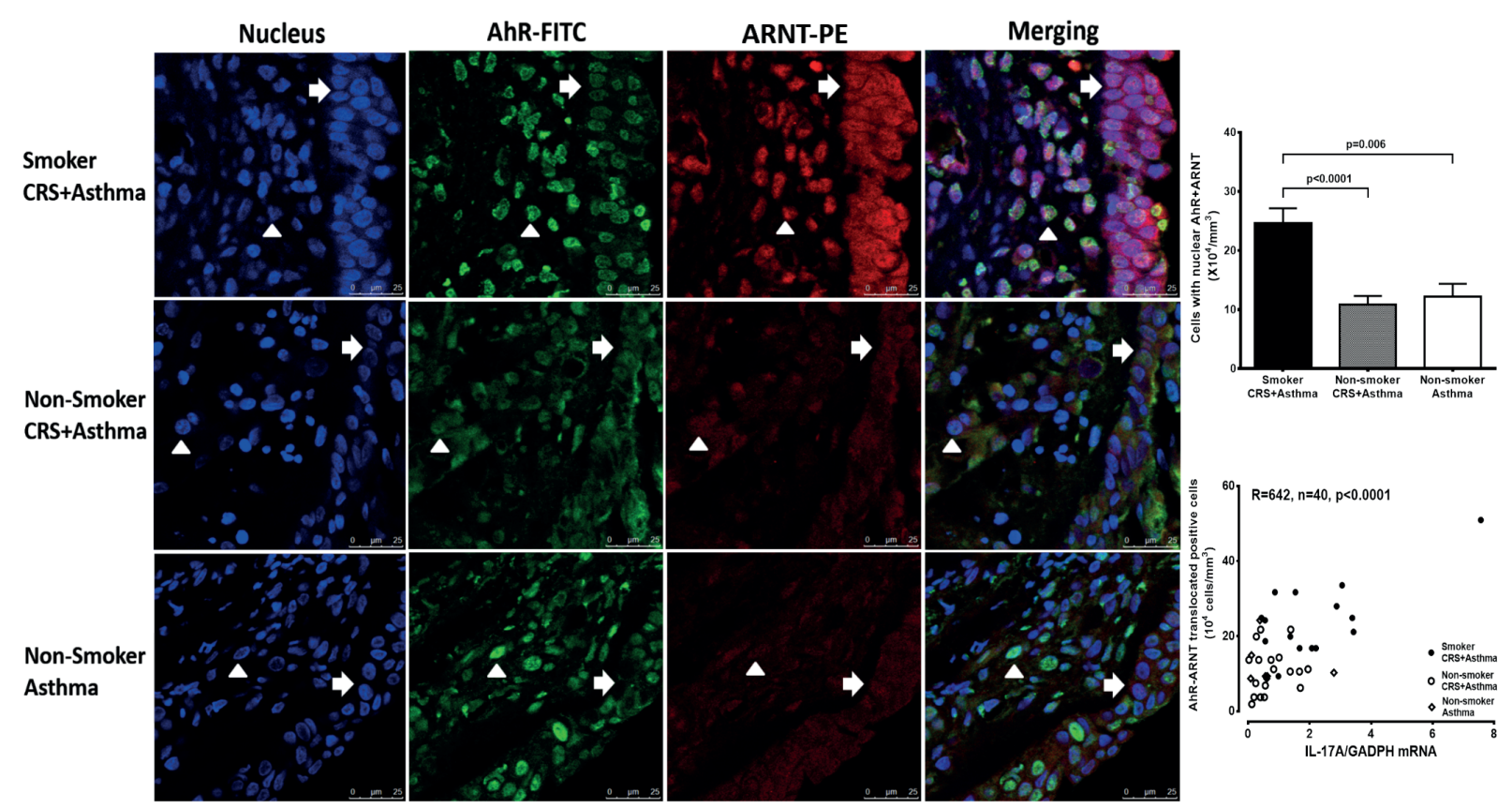

Figure 3. Confocal images showing AhR expression (green), ARNT expression (red), and nuclei (blue). The number of positively stained cells with nuclear AhR-ARNT complex (A, arrowhead) was higher in the nasal tissues from asthmatic patients with CRS who were smokers compared to asthmatic patients with CRS who were non-smokers, or asthmatic patients without CRS who were non-smokers (B). The number of nuclear AhR-ARNT translocated positive cells were strongly associated with the level of IL-17A mRNA in nasal tissues (C). Significance is indicated. Arrow: epithelium. Magnification: 400X. 


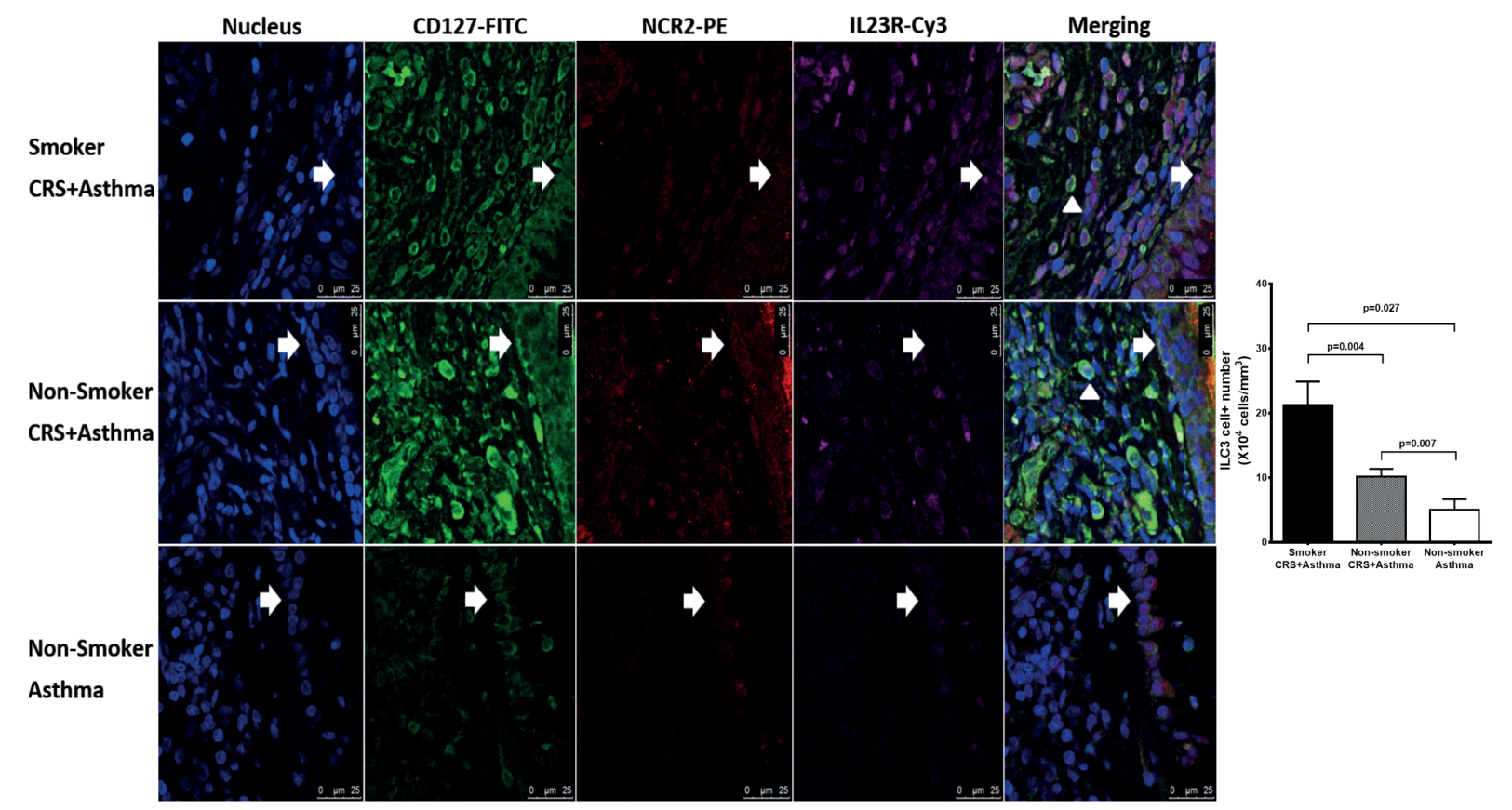

Figure 4. Confocal images of ILC3 cells with simultaneous immunostaining for CD127 (green), NCR2 (red), IL-23R (purple) and nuclei (blue). The cell numbers of NCR+ILC3 (arrowhead) were significantly increased in the nasal tissues derived from asthmatic patients with CRS who were smokers compared to asthmatic patients with CRS who were non-smokers or asthmatic patients without CRS who were non-smokers. Significance is indicated. Arrow: epithelium. Magnification: 400X.

A

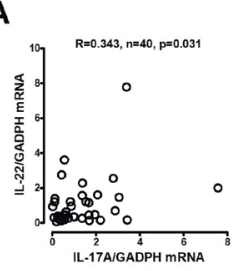

D

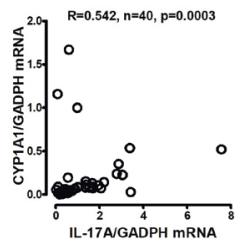

B

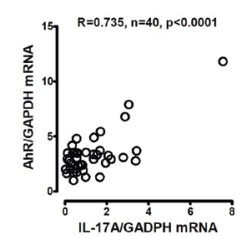

E

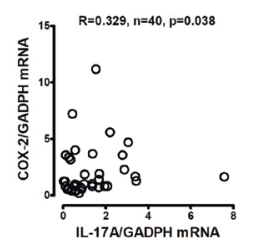

C

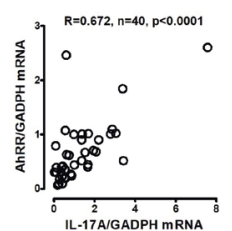

$\mathbf{F}$

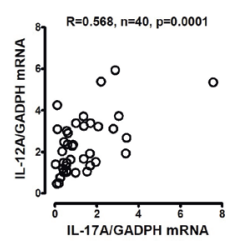

Figure 5. The mRNA levels of IL-17A had a significantly strong correlation with those of AhR (B), AhRR (C), CYP1A1 (D) and IL-12A (F) in nasal tissues. There were weak associations between the IL-17 mRNA expression and the mRNA expression of IL-22 (A) or COX-2 (E) in nasal tissues. The number and significance are indicated.

(Figure 3C).

Natural cytotoxicity receptor $\left(\mathrm{NCR}^{+}\right)$ILC3s, which were detected via simultaneous immunostaining for CD127, NCR2, and IL-23R, were significantly increased in the nasal tissues of smokers with asthma and CRS $\left(21.3 \pm 3.6 \times 10^{4}\right.$ cells $\left./ \mathrm{mm}^{3}, \mathrm{n}=15\right)$ than that of non-smokers with asthma and CRS (10.2 $\pm 1.2 \times 10^{4}$ cells $/ \mathrm{mm}^{3}$,

$\mathrm{n}=18, \mathrm{p}=0.004)$ and non-smokers with asthma without CRS (5.1

$\pm 1.6 \times 10^{4}$ cells $/ \mathrm{mm}^{3}, \mathrm{n}=7, \mathrm{p}=0.007$ ) (Figure 4).

\section{Correlation of IL-17A and ILC3 cells}

IL-17A mRNA expression level was strongly correlated with those of AhR, AhRR, and CYP1A1. Besides, there was a weak association between mRNA expression of IL-22 or COX-2 and IL-17A (Figure 5). However, the cell numbers of (NCR+) ILC3 had a strong association with IL-17A and COX-2 mRNA expression in nasal tissues. The cell numbers of $\left(\mathrm{NCR}^{+}\right)$ILC3 were weakly correlated with the mRNA expression of IL-22, AhR, and AhRR in the nasal tissues (Figure 6).

\section{Discussion}

This is the first study to simultaneously evaluate the impact of cigarette smoke on the airway inflammation in patients with asthma in terms of the corresponding clinical outcomes, IL-17A, $\mathrm{AhR}$, and (NCR+) ILC3 expression. Our results revealed that smokers had poorer improvement in asthma control, pulmonary function, and asthma treatment after nasal surgery, compared to those of non-smokers. The expression of AhR, $\left(\mathrm{NCR}^{+}\right)$ILC3, and IL-17A in the nasal tissues of asthmatic patients with CRS and cigarette smoking were elevated compared to asthma patients without CRS. The tissue level of IL-17A mRNA was correlated with AhR, AhRR, nuclear AhR-ARNT-positive cells and AhR target gene of CYP1 $A 1$, and its related-inflammatory mediators (COX-2). In addition, the cell numbers of $\left(\mathrm{NCR}^{+}\right)$ILC3 were cor- 
A

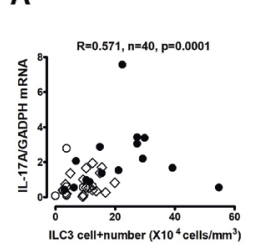

B

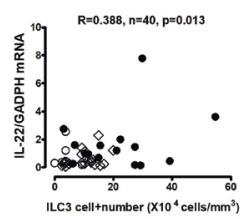

E

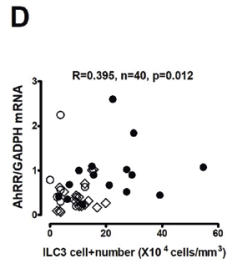

C

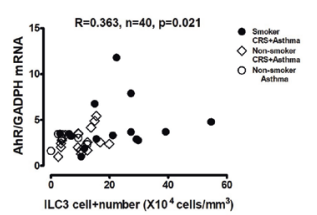

$\mathbf{F}$

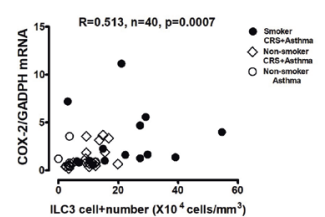

Figure 6. The cell numbers of $\left(\mathrm{NCR}^{+}\right)$ILC3 were significantly correlated with IL-17A (A), IL-22 (B), AhR (C), AhRR (D) and COX-2 (F) mRNA levels in nasal tissues, but not related to the mRNA level of CYP1A1. The numbers, correlation coefficients, and significance are indicated.

related with IL-17A and COX-2 mRNA expression and with IL-22, $A h R$, and AhRR in the nasal tissues. Therefore, cigarette smoking might initiate an IL-17A-mediated inflammation via activation of AhR and an increase in ILC3 cells in the airway mucosa of patients with asthma and CRS, resulting in an additional inflammatory effects on the original Th2-based airway inflammation, thus contributing to worse outcomes in asthma control after nasal surgery and being refractory to usual anti-asthma therapy. Cigarette smoke contains many AhR-activating ligands, which may contribute to AhR-dependent immune response in mucosal cells exposed to chronic cigarette smoke ${ }^{(23)}$ In this study, the expression of AhR, nuclear AhR-ARNT, CYP1A1, (NCR+) ILC3, IL-17A, IL-22, and COX-2 were elevated in the nasal mucosa of smokers with CRS and asthma. After binding to ligands, the AhR translocates to the nucleus and forms a heterodimer with the ARNT in the nasal tissue and has been shown to enhance the Th17 and ILC3 development ${ }^{(24)}$ and induce the expression of the cytokine IL-22 and IL-17A in these cells ${ }^{(28)}$. AhR may regulate transcription factors, cellular proteins, and responses to chemical compounds via the AhR-ARNT, NF-KB, and p38-MAPK signalling pathways ${ }^{(29)}$. A previous study showed that the elevated IL-17A expression in the lung explants from both non-COPD and COPD patients was dependent on the NF-KB pathways activated via exposure to cigarette smoke ${ }^{(30)}$. Smoke and other environmental stimuli act on airway epithelial cells to induce neutrophil chemotaxis along with neutrophilic inflammation of airways by producing IL-17A in asthmatic smokers ${ }^{(17)}$. Thus, the elevated IL-17A mRNA expression in the nasal tissues was correlated with those of AhR, AhRR, CYP1A1, and cell numbers with positive nuclear AhR-ARNT immunostaining. In addition, ILC3 cells, which express the transcription factors RORyt and AhR, have a high capacity to produce IL-17A and IL-22 ${ }^{(31)}$. AhR knock-out mice have reduced numbers

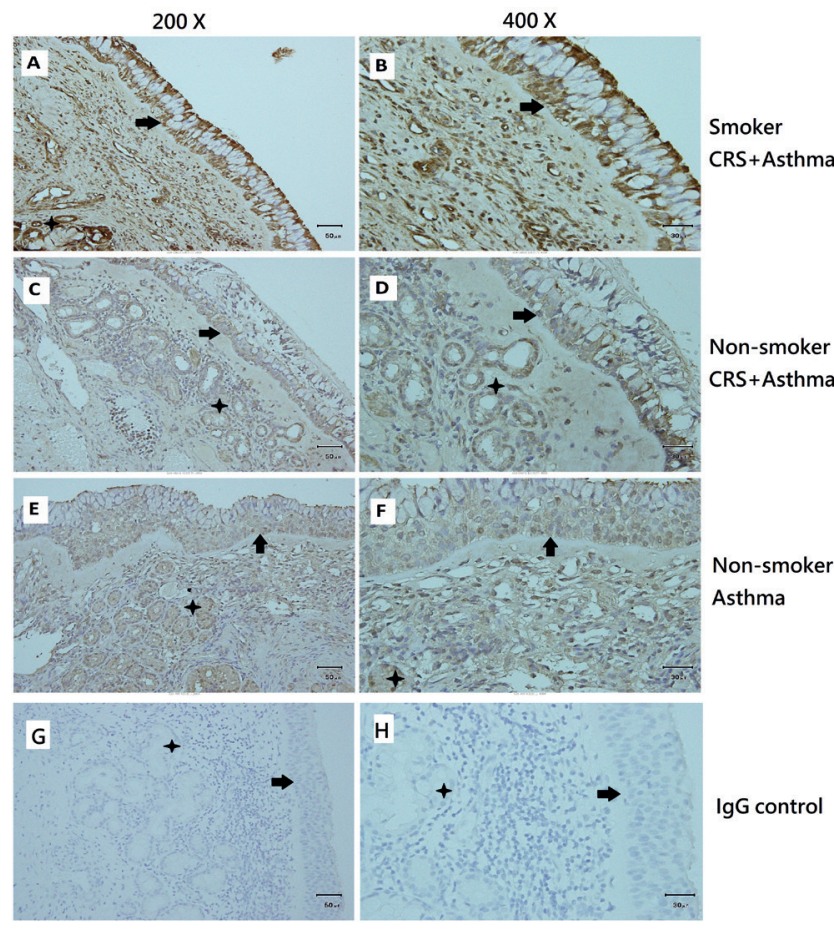

Figure S1. Immunohistochemistry study of IL-17A. The expression of $\mathrm{IL}-17 \mathrm{~A}$ in nasal tissues derived from asthmatic patients with CRS who were smokers ( $A$ and $B$ ) was stronger than that from asthmatic patients with CRS who were non-smokers ( $C$ and $D$ ) and asthmatic patients without CRS who were non-smokers (E and F). Arrow: epithelium, Asterisk: mucus gland.

of IL-22-producing ILC3 subset in the gut ${ }^{(31,32)}$. AhR activation plays an important role in the maintenance and function of IL-22-producing ILC3s in the gut ${ }^{(31)}$. (NCR+) ILC3 cells secrete IL-22 that highlights its critical role in activating the effector functions of committed Th17 cells, contributing to the development of inflammatory disease ${ }^{(33)}$. Therefore, the cell numbers of $\left(\mathrm{NCR}^{+}\right)$ ILC3 in the nasal tissues were correlated with the IL-17A, IL-22, $A h R$, and AhRR mRNA levels in the nasal tissues. The AhR-ARNT complex initiates the transcription of genes such as CYP1A1 that upregulated the downstream enzyme of COX-2, which is involved in the airway inflammation. Taken together, PAHs in cigarette smoke initiated the IL-17A-associated immune response via AhR activation. This study showed that Th17 cells and (NCR $)$ ILC3, while activated, contributed to the production of IL-17A and AhR translocation-induced cytokines, such as COX-2 and CYP1A1, among smokers with asthma and CRS.

Based on the united airways concept, CRS and asthma are viewed as two manifestations of a single pathological process that occur in different segments of the airway. Inflammation of the lower airways is influenced by the upper airway IL-17A expression in smokers with asthma and CRS through the systemic response of inflammatory mediators and/or aspiration of inflammatory sinus secretions ${ }^{(34)}$. Additionally, previous studies 
demonstrated that inflammatory responses of nasal epithelial cells could reflect those of bronchial cells, and therefore, nasal epithelial cells could be used as surrogates for lower airway cells $s^{(35)}$. Hence, we considered the same situation of high IL-17A secretion in the lower airways of smokers.

IL-17A increases the neutrophil-associated cytokines, CXCL8, IL$1 \beta, I L-6$, and GM-CSF from the airway epithelial cells, endothelial cells, and fibroblasts to mediate neutrophilic inflammation ${ }^{(31,36)}$. An IL-17A-related neutrophilic airway inflammation serves as a phenotype of severe asthma, mainly a steroid-insensitive Th2low phenotype ${ }^{(36)}$. Thus, IL-17A may be a key driver of disease exacerbation, resistance to corticosteroids, enhanced airway hyperresponsiveness, and increased airway remodeling in severe asthma ${ }^{(9,10,16,34)}$. Therefore, the increased expression of IL-17A in the nasal tissues of smokers who suffer from CRS and asthma appears to impair asthma control. In this and other studies, asthma control, including symptom scores, pulmonary function, steroid dependence, and emergency department visits, improve after an ESS treatment for CRS in patients with asthma ${ }^{(20,37)}$. However, this study further disclosed that the improvement in asthma control was less in patients who smoke cigarettes than those who do not smoke. Cigarette smoking seems to offset the benefit of nasal surgery, which is the reduction of inflammatory load in the airways.

Previous studies indicated that the induction of IL-17A via exposure to diesel exhaust particles, traffic-related air pollution, and PAH led to severe asthma ${ }^{(38)}$. Van Voorhis et al. demonstrated that the Th17 polarisation was enhanced in an AhR-dependent manner through the stimulation of atmospheric particulate matter (PM) in the lungs of mice. PAHs present in the PM were the likely sources of Th17-promoting activity ${ }^{(39)}$. Cigarette smoke contains substantial PAHs ${ }^{(22)}$. Taken together, IL-17A-mediated inflammation via AhR activation induced by cigarette smoke contributed to corticosteroid resistance and airway flow obstruction in patients with asthma.

IL-17A is primarily produced by Th17 cells ${ }^{(14,15)}$. Many immune cells, such as ILC3 $3^{(16)}, \mathrm{B}$ cells ${ }^{(40)}$, and $\gamma \delta T$ cells ${ }^{(41)}$ are also important cellular sources of IL-17A. In humans, the expression of NCR2, namely, NK cell activating receptor 44, defines two subpopulations among ILC3s ${ }^{(14)}$. (NCR+) ILC3s are scattered throughout the lamina propria of intestinal and airway mucosa ${ }^{(15)}$. However, (NCR-) ILC3 comprises lymphoid-tissue-inducer (LTi) cells and LTi-like cells that usually aggregate with stromal, dendritic, and B cells in the cryptopatches, isolated lymphoid follicles, or mature isolated lymphoid follicles ${ }^{(14,15)}$. ILCs in the intestinal tract and lower airways are well-studied ${ }^{(42)}$. However, few data about the relative abundance and characteristics of ILC3 cells in the human nasal mucosa are available ${ }^{(43)}$. This study showed that cigarette smoke may increase the number of $\left(\mathrm{NCR}^{+}\right)$ILC3 cells via AhR activation, which contributes to the production of IL-17A in the nasal mucosa of smokers with asthma and CRS.
Our study revealed that COX-2 was upregulated in the nasal tissues of smokers with asthma and CRS through AhR activation. The promoter activity of the COX-2 gene was induced by AhR ligands ${ }^{(44)}$, and this activation increased the binding of the AhR to XRE in the COX-2 promoter. Data revealed that COX-2 mRNA in smokers and non-smokers with asthma and CRS $(n=33)$ was correlated with the mRNA expression of AhR, AhRR, and CYP1A1 in the nasal tissues (data not shown). The recruitment of AhR to target promoter genes such as CYP1 A1 has been associated with the recruitment of transcription coactivators, such as NF$K B, N F-I L-6$, and $C / E B P \beta^{(45)}$, thus contributing to the production of COX-2. Smoking induces an array of inflammatory mediators and cytokines, such as COX-2, MMP-1, and MMP-9, and lead to chronic inflammation and airway remodeling in patients with asthma and rhinosinusitis. The poorer improvement of asthma control in smokers with asthma and CRS after nasal surgery may be partly related to this pathway.

In this study, poorer improvements in asthma control, pulmonary function, and asthma treatment, but not in nasal symptoms 6 months after nasal surgery for CRS, were observed in smokers than in non-smokers. Previous studies revealed that exposure to smoking diminishes long-term outcomes and increases the risk of revision surgery after $\operatorname{ESS}^{(3,7)}$. However, short-term outcomes of CRS after ESS in smokers was diverse as reported in the literature ${ }^{(46)}$. Rudmik et al. demonstrated a significant difference in the prevalence of worsening postoperative outcomes among heavy smokers, light smokers, and non-smokers, indicating that smoking volume may contribute to diverse results ${ }^{(6)}$. Smokers with asthma and CRS comprise a small population of patients with asthma, but have the highest health care costs among asthma phenotypes ${ }^{(9,26,47)}$. Therefore, defining the role of IL-17A and its associated immune pathway is imperative in designing, implementing, and tailoring therapeutics to successfully treat these patients. Future novel therapeutics that target IL-17A or AhR-related inflammatory responses or modulate the function of ILC3s may be beneficial for those with asthma or CRS that is refractory to current standard treatments.

This study has several limitations that warrant consideration. First, we recruited patients with asthma with and without CRS. It would have been better to include patients without airway diseases and CRS in the normal control group. However, it is difficult to recruit appropriate and healthy control patients because of the ethical considerations involved in performing an invasive biopsy on the nasal mucosa. Our ethics committee did not allow us to include this control group. Thus, non-smokers with asthma without CRS were chosen as control population. Second, the sample size was small. A large-scale study is necessary for the subgroup analysis of different phenotypes of CRS. Third, long-term studies are needed to investigate the continuing impact of cigarette smoking on the clinical outcomes of patients with CRS and asthma. Fourthly, we have not used flow 
cytometry which is an important method for the study of innate lymphoid cells (ILCS) isolated from tissue specimens that would have allowed for a multiparameter analysis of ILC3 and ILC2 populations. In the future, we will conduct the flow cytometry study to elucidate in the tissue cell suspensions of nasal mucosa these ILC3 and ILC2 populations.

\section{Conclusion}

Cigarette smoking was associated with the upregulation of AhR, (NCR $)$ ILC3, IL-17A, IL-22, and COX-2 in the nasal tissues of patients with airway inflammatory diseases and with a lesser improvement in asthma control after a nasal surgery. This contributes to the understanding of the possible mechanisms of inflammatory airway diseases initiated by environmental exposures, such as cigarette smoke. Future novel therapeutics that target IL-17A or AhR-related inflammatory responses or modulation of the function of ILC3s may be beneficial for those with asthma or CRS refractory to current standard treatments.

\section{Acknowledgements}

The authors received a research grant from the Chang Gung Memorial Hospital (CMRPG3D0831, CMRPG3G0671, and CMRPG3G0061). The funder had no role in the study design, data collection, and analysis; decision to publish; or preparation of the manuscript. The authors thank Ms. Meng-Chieh Tsai for helping in the data collection.

\section{Authorship contribution}

TJL and CHW designed conceptualized this study. Chi-Che Huang, PWW and PHC participated in the data collection. ChienChia Huang, CHF, HPK and CHW contributed to the data analysis and interpretation. Chien-Chia Huang wrote the original draft. TIL and CHW reviewed and edited the final manuscript.

\section{Conflict of interest}

The authors declare no conflict of interest.

\section{References}

1. Jarvis D, Newson R, Lotvall J, et al. Asthma in adults and its association with chronic rhinosinusitis: The GALEN survey in Europe. Allergy 2012;67(1):91-98

2. Lee $\mathrm{TJ}$, Fu $\mathrm{CH}$, Wang $\mathrm{CH}$, et al. Impact of chronic rhinosinusitis on severe asthma patients. PLoS One. 2017 15;12(2):e0171047.

3. Reh DD, Higgins TS, Smith TL. Impact of tobacco smoke on chronic rhinosinusitis: a review of the literature. Int Forum Allergy Rhinol 2012:2:362-369.

4. Hur K, Liang J, Lin SY. The role of secondhand smoke in sinusitis: a systematic review. Int Forum Allergy Rhinol 2014;4:2228.

5. Lee WH, Hong SN, Kim HJ, et al. Effects of cigarette smoking on rhinologic diseases: Korean National Health and Nutrition Examination Survey 2008-2011. Int Forum Allergy Rhinol 2015;5(10):937-943.

6. Rudmik L, Mace JC, Smith TL. Smoking and endoscopic sinus surgery: does smoking volume contribute to clinical outcome. Int Forum Allergy Rhinol. 2011; 1:145-152.

7. Krzeski A, Galewicz A, Chmielewski R, Kisiel $M$. Influence of cigarette smoking on endoscopic sinus surgery long-term outcomes. Rhinology. 2011 49(5):577-582.

8. Lambrecht BN, Hammad H. The immunology of asthma. Nat Immunol. 2015 ;16:45-56.

9. Akdis CA, Bachert C, Cingi $C$, et al. Endotypes and phenotypes of chronic rhinosinusitis: : a PRACTALL document of the European Academy of Allergy and Clinical Immunology and the American Academy of Allergy, Asthma \& Immunology. J Allergy Clin Immunol. 2013;131:1479-1490.

10. Newcomb DC, Peebles RS. Th17-mediated inflammation in asthma. Curr Opin Immunol. 2013;25:10

11. Liu Y, Zeng M, Liu Z. Th17 response and its regulation in inflammatory upper airway diseases. Clin Exp Allergy. 2015; 45:602-612.

12. Cao PP, Li HB, Wang BF, et al. Distinct immunopathologic characteristics of various types of chronic rhinosinusitis in adult Chinese. J Allergy Clin Immunol. 2009. 124:478-484

13. Wolk K, Witte E, Warszawska K, et al. The Th17cytokine IL-22 induces IL-20 production in keratinocytes: a novel immunological cascade with potential relevance in psoriasis. Eur J Immunol 2009; 39: 3570-3581.

14. Melo-Gonzalez F, Hepworth MR. Functional and phenotypic heterogeneity of group 3 innate lymphoid cells. Immunology. 2017; 150:265-275

15. Klose CS, Artis D. Innate lymphoid cells as regulators of immunity, inflammation and tissue homeostasis. Nat Immunol. 2016. 17:765-774.

16. Kim HY, Lee HJ, Chang YJ, et al. Interleukin17-producing innate lymphoid cells and the NLRP3 inflammasome facilitate obesityassociated airway hyperreactivity. Nat Med 2014; 20:54-61.

17. Siew LQC, Wu SY, Ying S, Corrigan CJ. Cigarette smoking increases bronchial mucosal IL-17A expression in asthmatics, which acts in concert with environmental aeroallergens to engender neutrophilic inflammation. Clin Exp Allergy 2017:47:740750.

18. Huang $\mathrm{CC}$, Wang $\mathrm{CH}, \mathrm{Fu} \mathrm{CH}$, et al, Association between cigarette smoking and interleukin-17A expression in nasal tissues of patients with chronic rhinosinusitis and asthma. Medicine (Baltimore). 2016; 95:e5432

19. Rowlands JC, Gustafsson JA Aryl hydrocarbon receptor-mediated signal transduction. Crit Rev Toxicol 1997; 27:109-134.

20. Nguyen LP, Bradfield CA The search for endogenous activators of the aryl hydrocarbon receptor. Chem Res Toxicol 2008; 21:102-116.

21. Profita M, Sala A, Bonanno A, et al. Chronic obstructive pulmonary disease and neutrophil infiltration: role of cigarette smoke and cyclooxygenase products. Am J Physiol Lung Cell Mol Physiol. 2010; 298:L261-L269.

22. Ranjit S, Midde NM, Sinha N, et al. Effect of polyaryl hydrocarbons on cytotoxicity in monocytic cells: potential role of cytochromes p450 and oxidative stress pathways. PLoS One. 2016; 11:e0163827

23. Kasai, A., Hiramatsu, N., Hayakawa, K. et al. High levels of dioxin-like potential in cigarette smoke evidenced by in vitro and in vivo biosensing. Cancer Res. 2006; 66: 7143-7150.

24. Quintana FJ, Basso AS, Iglesias AH, et al. Control of Treg and Th17 cell differentiation by the aryl hydrocarbon receptor. Nature 2008:453:65-71.

25. Baricza E, Tamási V, Marton N, Buzás El, Nagy G. The emerging role of aryl hydrocarbon receptor in the activation and differentiation of Th17 cells. Cell Mol Life Sci 2016;73:95-117.

26. From the Global Strategy for Asthma Management and Prevention, Global Initiative for Asthma (GINA) 2014. Available from: http://www.ginasthma.org/.

27. Fokkens WJ, Lund VJ, Mullol J, et al. EPOS 2012: European position paper on rhinosinusitis and nasal polyps 2012. A summary for otorhinolaryngologists. Rhinology 2012; 50:1-12.

28. Veldhoen M, Hirota K, Westendorf AM, et al. The aryl hydrocarbon receptor links TH17cell-mediated autoimmunity to environmental toxins. Nature 2008; 453: 106-109.

29. Nguyen NT, Hanieh $H$, Nakahama T, Kishimoto T. The roles of aryl hydrocar- 
bon receptor in immune responses. Int Immunol 2013; 25:335-343.

30. Chang Y, Al-Alwan L, Alshakfa S, et al. Upregulation of IL-17A/F from human lung tissue explants with cigarette smoke exposure: implications for COPD. Respir Res 2014;15:145.

31. Kiss EA, Vonarbourg C, Kopfmann S, et al. A. Natural aryl hydrocarbon receptor ligands control organogenesis of intestinal lymphoid follicles. Science 2011; 334:15611565.

32. Qiu J, Heller JJ, Guo X, et al. The aryl hydrocarbon receptor regulates gut immunity through modulation of innate lymphoid cells. Immunity 2012;36:92-104.

33. Sano T, Huang W, Hall JA, et al. An IL-23R/ IL-22 circuit regulates epithelial serum amyloid A to promote local effector Th17 responses, Cell. 2015; 163: 381-393.

34. Dixon AE, Kaminsky DA, Holbrook JT, Wise RA, Shade DM, Irvin CG. Allergic rhinitis and sinusitis in asthma: differential effects on symptoms and pulmonary function. Chest. 2006; 130:429-435.

35. McDougall CM, Blaylock MG, Douglas JG, et al. Nasal Epithelial Cells as Surrogates for Bronchial Epithelial Cells in Airway Inflammation Studies. Am J Respir Cell Mol Biol. 2008;39(5):560-8.

36. Chesne J, Braza F, Mahay G, Brouard S, Aronica M, Magnan A. IL-17 in severe asthma. Where do we stand? Am J Respir Crit Care Med 2014;190:1094-1101.
37. Schlosser RJ, Smith TL, Mace J, Soler ZM Asthma quality of life and control after sinus surgery in patients with chronic rhinosinusitis. Allergy 2017; 72:483-491.

38. Brandt EB, Kovacic MB, Lee GB, et al. Diesel exhaust particle induction of IL-17A contributes to severe asthma. J Allergy Clin Immunol 2013;132:1194-1204

39. van Voorhis M, Knopp S, Julliard W, et al. Exposure to atmospheric particulate matter enhances Th17 polarization through the aryl hydrocarbon receptor. PLoS One. 2013;8:e82545.

40. Vazquez-Tello A, Halwani R, Li R, et al. IL-17A and IL-17F expression in B lymphocytes. Int Arch Allergy Immunol 2012;157:406-416.

41. Murdoch JR, Lloyd CM. Resolution of allergic airway inflammation and airway hyperreactivity is mediated by IL-17-producing $\gamma \delta T$ cells. Am J Respir Crit Care Med 2010;182:464-476.

42. Montaldo E, Juelke K, Romagnani C. Group 3 innate lymphoid cells (ILC3s): Origin, differentiation, and plasticity in humans and mice. Eur J Immunol. 2015; 45:2171-2182.

43. Bal SM, Bernink JH, Nagasawa M, et al. IL-1 $\beta$, IL-4 and IL-12 control the fate of group 2 innate lymphoid cells in human airway inflammation in the lungs. Nat Immunol. 2016 Jun;17(6):636-645.

44. Degner SC, Kemp MQ, Hockings JK Romagnolo DF. Cyclooxygenase-2 promoter activation by the aromatic hydrocarbon receptor in breast cancer MCF-7 cells: repressive effects of conjugated linoleic acid. Nutr Cancer. 2007:59:248-257.

45. Smith WL, Dewitt DL, Garavito RM Cyclooxygenases: tructural, cellular, and molecular biology. Annu Rev Biochem 2000; 69:145-182.

46. Das S, Becker AM, Perakis H, Prosser JD, Kountakis SE. The effects of smoking on short-term quality of life outcomes in sinus surgery. Laryngoscope. 2007 117(12):22292232

47. Sorbello V, Ciprandi G, Di Stefano A et al. Nasal IL-17F is related to bronchial IL-17F/neutrophilia and exacerbations in stable atopic severe asthma. Allergy. 2015;70(2):236-240.

Professor Chun-Hua Wang, MD

Department of Thoracic Medicine

Chang Gung Memorial Hospital and

Chang Gung University, Taoyuan,

Taiwan

No. 5, Fu-Shin Street, Kweishan

Taoyuan 333, Taiwan

Tel: +886-3-3281200 ext. 8466

Fax: +886-3-3979361

E-mail:wchunhua@ms7.hinet.net 